\title{
Measuring quality of life after esophagectomy: Do we really need to go there?
}

\author{
Kenneth A. Kesler, MD
}

\author{
From the Thoracic Division, Department of Surgery, Indiana University, Indianapolis, Ind. \\ Disclosers: Author has nothing to disclose with regard to commercial support. \\ Received for publication Aug 28, 2015; accepted for publication Aug 31, 2015; available ahead of print Sept 26, \\ 2015. \\ Address for reprints: Kenneth A. Kesler, MD, Thoracic Division, Department of Surgery, Indiana University, \\ Barnhill Dr, EM \#212, Indianapolis, IN 46202 (E-mail: kkesler@iupui.edu). \\ $0022-5223 / \$ 36.00$ \\ J Thorac Cardiovasc Surg 2016;151:631-2 \\ Copyright (c) 2016 by The American Association for Thoracic Surgery \\ http://dx.doi.org/10.1016/j.jtcvs.2015.08.111
}

Esophagectomy is a large, complex operation usually performed for malignancy and like any other complex operation performed for cancer, precise execution of several sequential steps is critical for uneventful healing and recovery, not to mention good oncologic outcome. Also like most large, complex operations, success has been measured in terms of mortality rates. Although the incidence of esophageal adenocarcinoma has risen over the past 3 decades, esophagectomy mortality has proportionally decreased to acceptable levels in many centers over this same period of time. Unlike most other large gastrointestinal cancer operations, restoring gastrointestinal function is additionally necessary in esophagectomy and requires performance of several critical steps. But overall and disease-specific quality of life has not been well studied nor have standard benchmarks been established. Because a significant number of patients with esophageal cancer are living longer as a result of early detection and multimodal therapy, optimizing quality of life is increasingly important.

Esophagectomy can be performed by a number of different approaches and various replacement conduits can be constructed and routed using a variety of methods. Far and away the most commonly used replacement conduit for the esophagus is the stomach. Although reliable for healing, the stomach is a passive conduit dependent on gravity for drainage. Gastric conduit construction, including diameter, length, and orientation, can all significantly affect upper gastrointestinal tract function. Ingested food and liquid have potential to hang up in 3 locations: the esophagogastric anastomosis, stomach body, and gastric outlet. Poor conduit function (function is a bit of an oxymoron in the case of a passive conduit) is not infrequently a result of technical issues in any 1 or all 3 locations. Poor conduit empting also leads to more reflux, which negatively affects quality of life and can even result in aspiration tendencies with lifethreatening potential.

Adverse overall and disease-specific quality of life has been closely linked to complications following esophagectomy. ${ }^{1}$ For example, anastomotic leak not only represents a

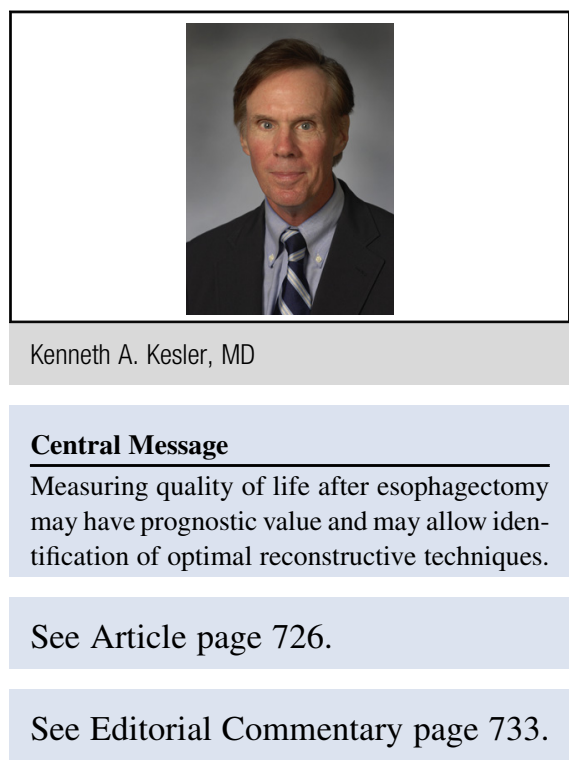

significant source of morbidity and mortality, but also not infrequently leads to anastomotic stricture. Anastomotic strictures may significantly negatively affect disease-specific quality of life and additionally increases cost of care when dilatation is required. ${ }^{2}$ Recent studies have also suggested a reduction in long-term survival in patients treated for anastomotic leaks. ${ }^{3}$ Risk factors for anastomotic leak and stricture are typically multifactorial in nature; however, technical issues such as a narrow diameter anastomosis and/ or an anastomosis with poor vascular supply are 2 major contributing factors.

Poor or declining quality of life after esophagectomy has been shown to be potentially predictive of decreased survival. Djarv and Lagergren ${ }^{4}$ found low health-related quality of life scores at 6 months following surgery to be independently predictive of mortality. Quality-of-life surveys administered to patients undergoing esophagectomy after induction chemoradiation therapy on a prospective protocol found a decrease in quality of life in the subset of patients who died within 1 year of surgery. ${ }^{5}$ Speculation includes occult or demonstrable recurrent disease as contributing to quality of life decline in these studies. A large national database found symptom clusters of reflux/ cough and eating difficulties significantly increased the risk of long-term mortality after esophagectomy. ${ }^{6}$ Although further study is needed to identify specific etiologies for poor overall and disease-specific quality of life, it seems likely that technical issues may play a not insignificant role. 
In addition to the normally needed resources, challenges to measure overall and disease-specific quality of life after esophagectomy are many. Foremost, commonality in quality-of-life measurement instruments will be important. Because quality of life is a subjective outcome, expectations before assessment may influence self-assessment. For example, a patient expecting to have completely normal quality of life after esophagectomy may report poor quality of life, whereas a patient prepared for poor quality of life after esophagectomy may believe he or she is doing well. Establishing a baseline disease-specific quality of life after an illness occurs, which in the case of esophageal cancer frequently results in dysphagia and weight loss, is a challenge. Patients experiencing toxicity during induction therapy typically require longer recovery times, making baseline and comparative quality-of-life assessment difficult. Finally, controlling for surgeon preference and experience in these large, complex operations will not be easy. Conversely, measuring esophageal-specific quality of life using a consistent surgical approach to establish baselines seems to represent a feasible starting methodology.

Because the vast majority of esophagectomies are performed for life-threatening disease, patients have been satisfied to simply survive. And surgeons are to be congratulated for lowering operative mortality and morbidity of these operations to acceptable levels. We should not stand complacent and neglect working to improve postoperative quality of life. Similar to patients where a suboptimal oncologic approach may lead to early cancer recurrence, a patient with a recurring anastomotic stricture or poorly emptying conduit resulting in adverse quality of life will likely not care the location, number, or size of incisions used during the operation and additionally may well be susceptible to early mortality not due to cancer. This subset of patients undergoing esophagectomy who have poor upper gastrointestinal tract function is, in no small part, responsible for giving esophagectomy a bad reputation. This reputation is worsened if the patient is not cured of the disease. Although the challenges are many, until we can more consistently optimize postoperative quality of life, some patients - such as patients at high surgical risk or patients with very locally advanced cancers and associated poor prognoses-may opt for nonsurgical treatment.

\section{CONCLUSIONS}

Serial global quality-of-life assessments may be a valuable prognostic tool. As the mortality related to esophagectomy has decreased, measuring disease-specific quality of life with adaption of surgical techniques that optimize upper gastrointestinal tract function is the next frontier. We hope the challenge will be accepted and quality-of-life benchmarks can be established.

\section{References}

1. Derogar M, Orsini N, Sadr-Azodi O, Lagergren P. Influence of major postoperative complications on health-related quality of life among long-term survivors of esophageal cancer surgery. J Clin Oncol. 2012;30:1615-9.

2. Price TN, Nichols FC, Harmsen WS, Allen MS, Cassivi SD, Wigle DA, et al. A comprehensive review of anastomotic technique in 432 esophagectomies. Ann Thorac Surg. 2013;95:1154-61.

3. Kofoed SC, Calatayud D, Jensen LS, Helgstrand F, Achiam MP, De Heer P, et al. Intrathoracic anastomotic leakage after gastroesophageal cancer resection is associated with increased risk of recurrence. J Thorac Cardiovasc Surg. 2015;150: 42-8.

4. Djarv T, Lagergren P. Six-month post-operative quality of life predicts long term survival after oesophageal cancer. Eur J Cancer. 2011;47:530-5.

5. Safieddine N, Zu Q, Quadri SM, Knoxx JJ, Hornby J, Sulman J, et al. Health related quality of life in esophageal cancer: effect of neoadjuvant chemoradiation therapy followed by surgical intervention. J Thorac Cardiovasc Surg. 2009;137: $36-42$

6. Wikman A, Johar A, Lagergren P. Presence of symptom clusters in surgically treated patients with esophageal cancer. Cancer. 2014;120:286-93. 